\title{
Hybrid laser information improved PSO-RBPF algorithm
}

\author{
Haibo Lin ${ }^{1,}$, Jingjing $\mathrm{Ke}^{1, \mathrm{~b}}$, Yi Zhang ${ }^{1, \mathrm{c}}$
}

'Engineering Research \& Development Center of Information Accessibility, Chongqing

University of Posts and Telecommunications, Chongqing 400065, China

a 316782900@qq.com, bkjj88212@163.com, ${ }^{c} 2215556374 @ q q . c o m$

Corresponding author: KE Jing-Jing, E-mail:316782900@qq.com

Keywords: Simultaneous localization and mapping (SLAM), Rao-Blackwellized particle filter (RBPF), particle swarm optimization (PSO), Robot operating system (ROS).

\begin{abstract}
To solve the estimator's inconsistency in calculating the proposal distribution and the particles degeneracy phenomenon of the Rao-Blackwellized particle filter (RBPF), an improved method of RBPF based on fusing Precision laser pre-observation information particle swarm optimization (PSO) is proposed to solve simultaneous localization and mapping (SLAM) problem of mobile robot. Compared with the traditional RBPF, the improved method fuses the robot's odometer and laser sensor information to proposal distribution computing, Meanwhile, the resampling process particle degeneration is applied particle swarm optimization(PSO) strategy to further optimize and adjust the obtained particle sets, The improved algorithm maintains the diversity of the particles and improves the consistency of robot's pose estimation effectively. The paper also evaluates the proposed method with the Robot Operating System (ROS), runs on platforms Pioneer3-DX robot which equipping with a URG laser sensors. The experiment results show that this method has realized online real-time high-precision grid map mapping successfully.
\end{abstract}

\section{Introduction}

Simultaneous localization and map building for mobile robot (SLAM) is a process that a robot relying on its onboard sensors builds environment map in an unknown environment, and determines its position on the map [1]. SLAM is a complex issue and needs to find a calculation method in high dimensional space, which combines the robot localization and mapping estimates. RaoBlackwellized particle filter (RBPF) proposed by Murphy, Doucet [2] and so on is an effective method to solve the problem of mobile robot SLAM, compared with the traditional Kalman and Markov algorithm, it has specific advantages which can effectively deal with nonlinear and nonGaussian systems without limiting to linear and Gaussian noise assumption.

Particle Swarm Optimization (PSO) algorithm is an emerging computing technology based on swarm intelligence theory. PSO has the best location of memory particles as well as the mechanisms for information sharing among the particles. PSO with unique particle memory can dynamically track current search to adjust search strategy [3].

\section{RBPF fundamentals of particle filter}

According to Murphy's RBPF algorithm, The essential idea of traditional RBPF-SLAM is linear decomposition of the state, to estimate map $m$ and robot path estimating $x_{1: t}=x_{1}, \cdots, x_{t}$ of the Joint posterior probability $p\left(x_{1: t}, m \mid z_{1: t}, u_{1: t-1}\right), z_{1: t}=z_{1}, \cdots, z_{t}$ which is observational data, odometer data $u_{1: t-1}=u_{1}, \cdots, u_{t-1}$. Recurrence formula for solving map $m$ in Eq.(1).

$p\left(x_{1: t}, m \mid z_{1: t}, u_{1: t-1}\right)=p\left(m \mid x_{1: t}, z_{1: t}\right) \bullet p\left(x_{1: t} \mid z_{1: t}, u_{1: t-1}\right)$

Eq. (1) we conclude that we shall estimate the trajectory of robots $p\left(x_{1: t} \mid z_{1: t}, u_{1: t-1}\right)$ at first and then according to the trajectory solving robot map $m$. Using the particle filter to estimate the trajectory of robots $p\left(x_{1: t} \mid z_{1: t}, u_{1: t-1}\right)$, each particle filter represents one possible path. 
The process of traditional RBPF particle filter is as follows [4, 5]:

1) Sampling the new robot pose: according to the proposed distribution of $\pi$, the nextgeneration particle collection $\left\{x_{t}^{(i)}\right\}$ is come from the previous generation $\left\{x_{t-1}^{(i)}\right\}$. Typically, the movement model of robot distributes as a proposal of sampling.

2) Calculating particle weight: in order to bridge the gap between targeted distribution and proposal distribution, you should calculate the weight of each individual particle $w_{t}^{(i)}$, by importance sampling formula:

$w_{t}^{(i)}=p\left(x_{1: t}^{(i)} \mid z_{1: t}, u_{1: t-1}\right) / \pi\left(x_{1 t t}^{(i)} \mid z_{1: t}, u_{1: t-1}\right)(i=1, \ldots, N)$

3) Re-sampling: the sampling operation is necessary because the proportion of particles are selected by their weight, particles with limited number are used to approximate a continuous distribution, and particle sampling proposal filters distributed $\pi$ have a gap with target distribution. After re-sampling, all particles have the same weight.

4) Map update: for each particle, you can use the track $x_{1: t}^{(i)}$ and observed information $z_{1: t}$ to calculate the corresponding $p\left(m^{(i)} \mid x_{1: t}^{(i)}, z_{1: t}\right)$, then update the map.

Currently traditional RBPF particles filter only used mileage meter movement model as proposed distribution, which needs large sampling particles. Frequently re-sampling may led to particle degeneration, and proposed distribution mostly affects the precision of estimates. Proposing the fusion laser observation information can improve the proposed distribution of RBPF, in order to avoid particle degeneration phenomenon in re-sampling and increase the diversity of particles set, introduced particles group optimization strategy into RBPF particles filter can solve the particles impoverishment in importance sampling. The improved method of RBPF-SLAM increased consistency and accuracy of the robot pose estimation while maintaining the diversity of the particle.

\section{Hybrid laser information improved PSO-RBPF}

\section{1 improving the prediction of particle distribution}

Map building conducted by mobile robot highly correlated with robot pose. Using particle filters calculate the current location, represented by $(x, y, \theta)$ in the Cartesian space. Particles predict the distribution of $q\left(x_{t} \mid x_{t-1}, z_{t}\right)=p\left(x_{k} \mid x_{k-1}\right)$, it obtained by the system evolution of $\hat{x}_{t}=f_{t}\left(x_{t-1}, u_{t}\right)$. The traditional distribution of particle filter ignores the function of the current observation $z_{t}$. herefore, this paper will be divided into the following three steps into to propose the observation of the laser sensor information [6]:

1) predict an intermediate state $\hat{x}_{t}$, by the last status of $x_{t-1}$ and the linear Gaussian motion model

$\hat{x}_{t}=f\left(x_{t-1}, u_{k}\right)$

$\hat{P}_{t}=\nabla f_{x} \hat{P}_{t-1} \nabla f_{x}^{\mathrm{T}}+Q$

Among them, $Q$ stand for laser rangefinder noise covariance matrix.

2) Treat Eq.(4) as an initial state of the extended Kalman filter, using it to update each observed characteristics, is described as Eq. (5) and Eq. (8)

$\hat{z}_{t}^{i}=h\left(\hat{x}_{t}, \theta_{t}^{i}\right)$

$P_{z_{t}} \cong \nabla h_{x} \hat{P}_{t} \nabla h_{x}^{\mathrm{T}}+\nabla h_{\theta} \hat{P}_{f} \nabla h_{\theta}^{\mathrm{T}}+R$

$K=\hat{P}_{t} \nabla f_{x}^{\mathrm{T}} \hat{P}_{z_{k}}$

$x_{t}=\hat{x}_{t}+K\left(z_{t}^{i}-\hat{z}_{t}^{i}\right)$

Among them, the $\nabla h_{\theta}, \nabla h_{x}$ is the Jacobin matrix of observation equation $h\left(x_{t}, \theta_{t}\right)$ respectively on

Observation State $\theta_{t}$ and State $x_{t}$.R is a covariance matrix of motion control model noise .

3) From the posterior probability distributions of the output result, the current entred observations of new particle distribution can be gained, see Eq. (10). 
$P_{t}=\hat{P}_{t}-K \nabla f_{t} \hat{P}_{t} ; \hat{P}_{t}=[0]$

$\hat{x}_{t}=\operatorname{drawSample}\left(x_{t}, P_{t}\right)$

\subsection{Particle Swarm Optimization Strategy}

Integration of laser observation information can make the proposal distribution closer to the true target distribution, re-sampling particle depletion phenomena still exist in the process. PSO [7] is used to optimize re-sampling particle iteration and adjust the sample set of particles in this paper.

1)Particle weight is calculated. RBPF-SLAM algorithm based on fusion laser projections information obtains a set of particles on behalf of robot pose. If $\left\{S_{i}\right\}_{i=1,2, \ldots, N}$ is a set of particles used to predict the state of the robot pose, the particle update formulas are

$$
\left\{\begin{array}{l}
V_{i}(t+1)=w V_{i}(t)+c_{1} r_{1}\left[S_{\text {pbest }}-S_{i}(t)\right]+ \\
\quad c_{2} r_{2}\left[S_{\text {gbest }}-S_{i}(t)\right], \\
S_{i}(t+1)=w V_{i}+\varepsilon S_{i}(t)
\end{array}\right.
$$

$S_{i}(t)$ means the position of particle $i$ at the time of $t ; S_{\text {pbest }}$ and $S_{g b e s t}$ are separately meant the optimal solutions of robot pose distribution locally and globally. Then using $\omega_{i}^{t}=\omega_{i}^{t} p\left(z^{t} \mid s^{i}, \lambda^{t}\right)$ calculates the weight of each particle, the eight minimum particle is $\left\{S_{l}, \omega_{l}\right\}$.

2) Particles option. Fitness function is applied between $F(\bullet)$ and particle attraction $F_{j, k}$ for energy efficiency,

$F\left(S_{i}\right)=\omega_{i}^{t}$

$F_{j, k}=\frac{F\left(S_{j}\right)-F\left(S_{k}\right)}{d_{j, k}}$

$d_{j, k}=\sqrt{\left(S_{j}-S_{k}\right)^{2}}$

In formula(13) $F_{j, k}$ means that particle $j$ has an energy efficiency appeal on particle $k$. In this paper $F_{j, k} \geq 0$ that means the particle $j$ attracted by particle $k$ or two particles in the energy balance. In formula (8), the other particles have the maximum energy efficiency of $F_{j, \max }$ attraction on particle $j$ at the time $t$. Let $S_{l}$ meets energy efficiency attracted function of particles, and expressed as the $\left\{S_{m}, \omega_{m}\right\}$.

$F_{j, \max }=\arg \max \left\{F_{j, n} \mid n=1,2, \ldots, N\right\}$

3) Particles replace. Mutation in genetic algorithm can optimize the particle set $\left\{S_{i}\right\}_{i=1,2, \ldots, N}$ for the following operations.

Define mutation as follows:

$$
\begin{aligned}
& \left\{\begin{array}{l}
S_{\text {new }}=(1-a) S_{l}+a S_{m}, \\
\omega_{\text {new }}=\omega_{l}+a\left(\omega_{m}-\omega_{l}\right)
\end{array}\right. \\
& P_{\text {mut }}=\omega_{l} / \omega_{m}
\end{aligned}
$$

With $P_{\text {mut }}$ probability for Genetic Variation, $\left\{S_{\text {new }}, \omega_{\text {new }}\right\}$ are variations of new particles. Using new particles $\left\{S_{\text {new }}, \omega_{\text {new }}\right\}$ substitute weight minimum particle $\left\{S_{l}, \omega_{l}\right\}$. Thereby improved the resampling process particles degeneracy.

\subsection{Hybrid laser information improved PSO-RBPF algorithm}

The steps as shown below:

Step1 according to $\mathbf{2 . 1 m e t h o d , ~ u p d a t e s ~ t h e ~ p r o p o s e d ~ d i s t r i b u t i o n ~ o f ~ p a r t i c l e , ~ i n i t i a l ~ p o s e ~}$ estimation $x_{t-1}^{i}$ for robot concludes the estimation on the initial position and odometer information;

Step2 Re-sampling: according to the algorithm in $\mathbf{2 . 2}$, repeatedly searching the obtained set of 
particles will get a repeated set of new particle search, until it meets the criteria. $N_{\text {eff }}=\frac{1}{\sum_{i=1}^{N}\left(\omega^{i}\right)^{2}} \geq N / 2$

Step3 according to the position of the robot $x_{t}^{i}$ and the observation information of $z_{t}$ calculate $m^{i}$.

\section{Hybrid laser information improved PSO-RBPF algorithm based on the ROS system realization}

\subsection{Introduction on test platform}

A platform ROS on is based on the Pioneer3-DX robot. Sensor uses Pioneer3-DX precise odometer and Hokuyo laser rangefinder URG04. Pioneer3-DX and PC/ROS through USB/RS458 communicate, as shown in Fig.1. ROS [8, 9] is an operating system installed on the Linux operating system. ROS mainly concludes the package (packages), nodes (nodes), themes (topics), message (messages) and servers (services). A node that represents a specific function of the executable program can accept data from robot sensors; establish communication between nodes and nodes through messages. Messages publish through topic. Any node can subscribe to and publish message on the subject, and the associated nodes can be encapsulated in a bag.

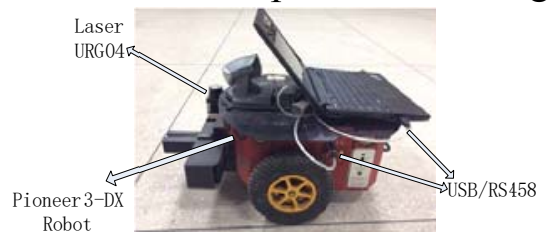

Fig. 1 experimental platform

\subsection{Software Implementation}

After building a good hardware platform, you need to write for Pioneer3-DX robot SLAM in unknown environment feature package. The main Nodes is as shown in Fig.2. The /base_link in this picture is a public Topic, in which all of Message interact. Odometer node, Laser Scan node are used to record and published mileage information, laser information into the Topic; rosaria node extraction Rosaria by publishing information on the Topic of information is used to control the Pionner3 robot. Master is used to register and find nodes; Map Building generates online maps in real time while extracting the mileage meter, laser information and improving algorithms.

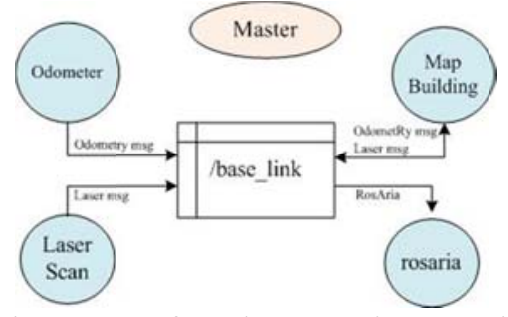

Fig. 2 The map of nodes running on the ROS

\subsection{ROS RBPF SLAM based on Particle Swarm Optimization to achieve}

This experiment carried out on Pioneer3-DX mobile robots. The robot is equipped with a URGHUKUYO laser range finder and a serial line to connect PC,PC for Linux (Ubuntu12.04) running on the ROS system via serial line to control the robot move, and odometer and laser online mapping and data display [10]. To complete the experiment, in turn, demand nodes:

\$ roscore; \$ rosrun ROSARIA rosaria ; \$ rosrun SLAM_mapping transform ; \$ rosrun SLAM_mapping mapping; \$ rosrun Rviz rviz.

Roscore is used to open the entire system. Rosaria nodes for the PC is used to interact with robots; ransform nodes transfer the laser coordinate orientation method and coordinate of mileage point into unified coordinate system; mapping node is the focus of this article, it will be based on the measure information of laser and odometer as well as the improved algorithm to complete the 
SLAM and finally pass the Rviz real time display. As shown in Fig.3, Off-white $\mathrm{w}$ is the test environment, black areas $\mathrm{B}$ is obstructions, $\mathrm{D}$ is laser scan points set and $\mathrm{P}$ is mobile robot mapping path.

This experiment uses traditional RBPF algorithm and algorithm for map building. Fig. 4 is a traditional RBPF 40 particles which is used to build the environment map, because traditional sampling algorithm from the model without considering the new observations, causing the error is large and the inconsistencies of building map (like a, b, c, d, e). Using the proposed algorithm takes only 10 particles that can build high-precision maps, as shown in Fig. 5.

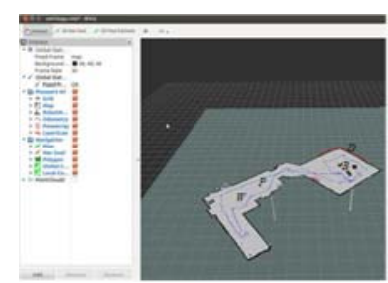

Fig.3 Real-time map on Rviz

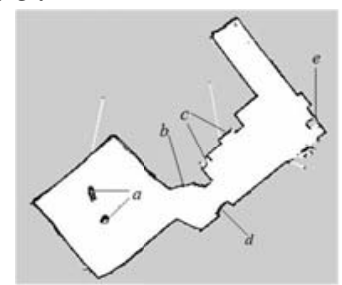

Fig.4 Map built by the traditional Fast SLAM algorithm

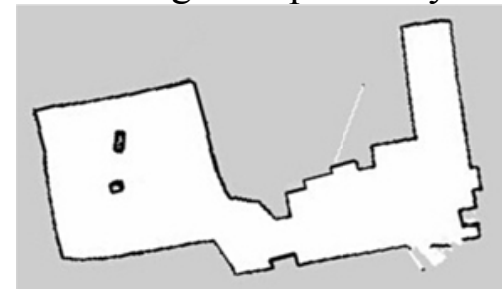

Fig. 5 Map built by the improved algorithm

\subsection{Analysis and Experimental results}

Table 1 compares the needed number of particles and the time when the traditional RBPF-SLAM algorithm and improved algorithm create the consistent maps. It can be seen that improved algorithm requires the number of particles much smaller than the traditional algorithm and also less time to create a map. You can see algorithm requires the number of particles much smaller than the traditional method, and also less time to create a map. Algorithm Uses the convergence of high precision laser information in the proposal distribution, at the same time introduced repeated particle swarm optimization strategy for particle drying up in the process of re-sampling. The method for improves the consistency and accuracy of the filter, and compared with the traditional RBPF method requires less number of particles. And the handling time decrease as the particle reduction, so the improved algorithm not only improves the efficiency and shorten the processing time.

Table 1 Parameters list of building a consistency map

\begin{tabular}{lcc}
\hline Algorithm & Number of particles & Elapsed time/s \\
RBPF-SLAM & 80 & 600 \\
Proposed algorithm by author & 10 & 225 \\
\hline
\end{tabular}

Table 2 includes the comparison of the mean, variance and error when using 5 particles in two algorithms.

Table 2 The estimation accuracy comparison of two kinds of algorithm

\begin{tabular}{cccc}
\hline \multirow{2}{*}{ Algorithm } & \multicolumn{2}{c}{ MSE } & \multirow{2}{*}{ errors } \\
& mean & variance & \\
\hline RBPF-SLAM & 0.783 & 0.500 & -0.6927 \\
Proposed algorithm by author & 0.083 & 0.076 & -0.4861 \\
\hline
\end{tabular}

\section{Conclusion}

On the base of the traditional RBPF-SLAM algorithm, incorporating the current observation information from the laser rangefinder, improving the predictive distribution functions of particle filter to make the sample particles closer to the actual distribution. Meanwhile, as for the problem of particle-code dried after re-sampling, this research imports Particle Swarm Optimization Strategy in order to reduce the appearance of the re-sampling, Experiments proved that this method can 
effectively maintain both the diversity of the particle and the consistency of the pose estimation of the robot as well as improve the robustness of SLAM. What's more, it can also achieve highprecision 2D raster map online under unknown environment.

\section{References}

[1]. Kim C, Sakthivel R, Chung W K. Unscented Fast SLAM: a robust and efficient solution to the SLAM problem [J]. IEEE Transactions on Robotics, 2008, 24(4): 808-820.

[2]. MONTEMERLO M, THRUN S, KOLLER D, et al. Fast SLAM: a factored solution to the Simultaneous localization and mapping problem[C]//Proceedings of the 18th National Conference on Artificial Intelligence. Cambridge, MA: MIT Press, 2002: 593-598.

[3]. Li Dalin, Li Jie .Task allocation method for multi-loitering units using PSO algorithm [J]. Transactions of Beijing Institute of Technology .2010, 30(12):1436-1439.

[4]. GRISETTI G, STACHNISS C, BURGARD W. Improved techniques for grid mapping with Rao-Blackwellized particle filters [J]. Robotics, IEEE Transactions, 2007, 23(1): 34-46.

[5]. Kim C, Kim H K, Chung W K. Exactly Rao-Blackwellized unscented particle filters for SLAM[C]/Proceedings of the 2011 IEEE International Conference on Robotics and Automation. Shanghai, China: IEEE, 2011, 3589-3594.

[6]. Zhang Yi, Cheng Teifeng, Luo Yuan, Fu Youli .Robust robot simultaneous localization and mapping implementation based on improved particle filter[J].Application Research of Computers,2015,32(8):2339-2342.

[7]. MA J C, ZHANG Q,XIE W, et al. Particle swarm optimization method based on mobile robot SLAM [J]. Transactions of Beijing Institute of Technology, 2013, 33(11):1151-1154.

[8]. ZHANG J W, ZHANG L X, HU Y, et al. The Open source robot operating system-ROS [M]. Beijing, Science Press, 2012. (in Chinese)

[9]. Aaron Martinez Enrique Fern á ndez. Learning ROS for Robotics Programming [M]. Beijing, China Machine Press, 2014

[10]. LUO Y, FU Y L, CHENG T F. Simultaneous localization and mapping implementation based on the improved Rao-Blackwellized particle filter[J]. Control Theory \& Applications, 2015, 32(2):267-272. (in Chinese) 